\title{
Effect of irrigation water salinity on the infectivity and reproduction of the root-knot and the reniform nematodes on some vegetable crops.
}

\author{
Al-Sayed A. Al-Sayed, Ahmed A. Farahat and Shaimaa F. Diab \\ Department of Zoology and Agric. Nematology, Fac. Of Agric. Cairo University.
}

\begin{abstract}
The influence of $\mathrm{NaCl}$ on the infectivity and reproduction of the root-knot and the reniform nematodes was studies using four concentrations $(500,1000,2000$ and $3000 \mathrm{ppm}$ ) of sodium chloride $(\mathrm{NaCl})$ in the irrigation water of eggplant, squash and cowpea. Increasing salt concentration and consequently soil Ec resulted in significant increase in number of $M$. incognita galls and egg-masses/ eggplant roots as compared with the check plants ( 0 salt). The highest number of galls was observed at $3000 \mathrm{ppm} \mathrm{NaCl}$. Increasing $\mathrm{NaCl}$ concentration in irrigation water increased females fecundity on the roots of eggplant and squash. However opposite results were observed on cowpea roots. The reniform nematode behaved differently, whereas the nematode counts increased with increasing salt concentration on cowpea and decreased on eggplant and squash.

The present results indicated that higher salt concentration adversely affected the growth of treated plants and significantly increased the damage caused by nematodes. The influence of salt stress on the nematode development and reproduction depend on nematode species, salt concentration (soil EC) as well as the host plant. In general damage in plants under salt stress and infected with the reniform nematode was more pronounced than those infected with the root-knot nematode.
\end{abstract}

Key words: $M$. incognita, $R$. reniformis, salinity.

\section{Introduction}

Salinity of either soil or irrigation water has become one of the important abiotic factors that affect agricultural production especially in countries suffering from shortage of their fresh water resources. In Egypt, the dependence on the ground water in the newly reclaimed sandy soil of the desert inflated the problem whereas soil salinity increased gradually year after year. Such increase in soil salinity adversely affected the growth of the cultivated field or fruit crops. In such kind of soil type, the sandy soil, nematodes also take part in the crop losses and intensifying the problem which may threat the sustainable agriculture. Positive correlations between citrus nematode populations and chloride content of soil water was observed by Kirkpatrick and Van Gundy 1966, Venkates and Das 1980, Youssef et al. 1989, Mashela et al. 1992. Different effects, increasing or 
decreasing nematode hatching, developing and reproduction have been observed with different mineral or salt concentrations on various nematode species (Jairajpuri et al. 1974, Lal and Yadav 1975, Edongali and Ferris 1980,1981, Ray and Das 1980, Edongali et al. 1982,Khan and Khan 1990and Sweelam 1994). Vermak et al. 2007 reported negative correlation between $M$. incognita galling, eggmasses, eggs/eggmass, final population and increasing saline conductivity. $R$. reniformis tolerated strongly saline conditions (Ray and Das 1980). No available recent references in literature illustrating the role of the gradual increase in soil salinity on plant nematodes dynamics. Accordingly this study was carried out to clarify such role under greenhouse conditions especially the root-knot, Meloidogyne incognita and the reniform, Rotylenchulus reniformis on three different host plants.

\section{Materials and methods}

The development and reproduction of the root-knot and the reniform nematodes under the stress of soil salinity was studied under greenhouse conditions of $30 \pm 5{ }^{\circ} \mathrm{C}$. Nematode cultures were belonged to the pure stock cultures of Nematology Research Center, Faculty of Agric. Cairo University. The study was carried out using 5 different salt concentrations (0, 500, 1000, 2000 and 3000 ppm) of sodium chloride $(\mathrm{NaCl})$. The solutions were used to irrigate eggplant, Solanum melongena (gloyana F1), squash, Cucurbita maxima (Tabark F1) and cowpea, Vigna unguiculata (cv Qaha1) grown in $15 \mathrm{~cm}$ diameter clay pots filled with loamy sand soil ( 1 sand : 1 loam v/v). Fifteen days after cultivation pots of each crop were divided into three groups and thinned to one plant/pot. Pots of the first group were inoculated with $2000 \mathrm{~J}_{2}$ of the root-knot nematode, M. incognita. Those of the second group were inoculated with 2000 infective stage of the reniform nematode, $R$. reniformis however pots of the third group were kept without inoculation to serve as checks. For each nematode species in each crop, 4 replicates were used in each salt concentration. Similar treatments were carried out with the uninoculated healthy plants, as well as 4 replicates were kept without any treatment to compare the results. Plants were irrigated every other day with $200 \mathrm{ml}$ of the prepared solutions, check treatments were irrigated with the same volume of tab water until the end of the experiment. Soil electrical conductivity (EC) was measured twice, after 22 days and at the end of the experimental time (Table 1) using Muliparameter analysis $(\mathrm{pH}$, EC and temperature) at the Reclamation \& development Center for Desert Soils, Soil Department, Fac. of Agric. Cairo University. At the end of the experimental time, plants were harvested, plant growth criteria were registered and soil and nematode populations were counted.

The obtained results were statistically analyzed according to the SPSS software package version 12 (SPSS, 2003). 
Table (1). Electrical conductivity of soils (1:2.5 soil extract) treated with different concentrations of $\mathrm{NaCl}$ after 22 days and at the end of the experiment.

\begin{tabular}{ccccccc}
\hline \multirow{2}{*}{$\begin{array}{c}\text { Salt concentration } \\
(\mathbf{p p m})\end{array}$} & \multicolumn{7}{c}{ EC mmhos/ cm } \\
\cline { 2 - 7 } & \multicolumn{2}{c}{ Eggplant } & \multicolumn{2}{c}{ Squash } & \multicolumn{2}{c}{ Cowpea } \\
\cline { 2 - 7 } & 22days & 45days & 22days & 45days & 22 days & 45 days \\
\hline $\mathbf{0}$ & 0.26 & 0.30 & 0.27 & 0.30 & 0.26 & 0.29 \\
$\mathbf{5 0 0}$ & 0.84 & 0.89 & 0.83 & 0.87 & 0.81 & 0.94 \\
$\mathbf{1 0 0 0}$ & 1.52 & 1.62 & 1.48 & 1.52 & 1.88 & 2.13 \\
$\mathbf{2 0 0 0}$ & 3.16 & 3.21 & 3.12 & 3.17 & 3.02 & 3.22 \\
$\mathbf{3 0 0 0}$ & 3.54 & 3.68 & 3.31 & 3.74 & 3.16 & 3.42 \\
\hline
\end{tabular}

\section{Results}

Data in table (2) indicated that increasing salt concentration and consequently soil ECs resulted in significant increase in the number of galls and egg-masses/ eggplant root as compared with the untreated plants (0 salt).

The highest number of galls was observed at $3000 \mathrm{ppm} \mathrm{NaCl}$ and varied significantly with other treatments. Almost similar results were observed with the number of galls/squash root but the highest significant number of galls was observed when squash was irrigated with 1000 ppm of sodium chloride solution. On cowpea, the opposite was observed, whereas, increasing salt concentration from 1000 to 3000 decreased significantly the observed numbers of galls and eggmasses as compared with 0 and 500 ppm. The lowest numbers of galls and eggmasses / cowpea roots were observed at the higher salt concentrations and varied significantly with the other treatments.

The number of eggs/egg-mass significantly increased with increasing salt in irrigation solutions in case of eggplant and squash, however, on cowpea roots the opposite was observed. The total number of eggs/pot and the number of eggs/g soil decreased with increasing salt concentration in case of squash and cowpea and increased in case of eggplant. Finally, the highest rate of build up was observed at $2000 \mathrm{ppm}$ of $\mathrm{NaCl}$ on eggplant and squash (EC 3.21, 3.17, respectively) and at 1000 ppm on cowpea (EC 2.13). With the reniform nematode, negligible numbers of developmental stages and mature females were counted on the roots of the three host plants, so no obvious trend could be observed due to salt concentrations (Table 3). Numbers of egg-masses significantly decreased at 1000, 2000 ppm on eggplant, at all concentrations on squash and at higher concentrations on cowpea as compared with the check. The number of eggs laid in the egg-masses on eggplant roots were not affected by different $\mathrm{NaCl}$ concentrations. While, it significantly decreased by all concentrations on squash and by only the highest concentration on cowpea as compared with the check. Consequently, the number of eggs/g soil and the total number of eggs/ pot behaved the same. 
Table (2): Development and reproduction of $M$. incognita on some vegetable orops as affected by different concentrations of sodium chloride.

\begin{tabular}{|c|c|c|c|c|c|c|c|c|c|}
\hline \multirow{2}{*}{ Vegetable crop } & \multirow{2}{*}{$\begin{array}{c}\text { Sait } \\
\text { concentration }\end{array}$} & \multicolumn{8}{|c|}{ Nema tode counts } \\
\hline & & $\begin{array}{l}\text { No. galls l } \\
\text { plant }\end{array}$ & $\underset{\text { I plant }}{\text { Eggmas } 595}$ & $\begin{array}{c}\text { Eggs/ } \\
\text { eggmass }\end{array}$ & $\begin{array}{l}\text { Total no. } \\
\text { өggs }\end{array}$ & $\begin{array}{c}\text { Eggs/g } \\
\mathbf{5 0 l l l}\end{array}$ & $\begin{array}{c}\text { soll } \\
\text { population }\end{array}$ & $\begin{array}{c}\text { Final } \\
\text { population }\end{array}$ & PtPI \\
\hline \multirow{5}{*}{$\begin{array}{l}\text { Eggplant } \\
\text { (Gloyana F1) }\end{array}$} & 0 & 134.80 & $76.3 d$ & $158.8 \mathrm{~d}$ & 12104.70 & 6.10 & 0.0 & 76.30 & $0.04 \mathrm{c}$ \\
\hline & 500 & $257.8 \mathrm{~b}$ & $129.8 \mathrm{C}$ & $241.3 \mathrm{~b}$ & $313022 \mathrm{C}$ & $15.7 \mathrm{C}$ & 0.0 & $129.8 \mathrm{C}$ & 0.050 \\
\hline & 1000 & $165.3 \mathrm{c}$ & $163.8 \mathrm{~b}$ & $201.0 \mathrm{C}$ & $32913.8 \mathrm{C}$ & $16.5 \mathrm{c}$ & 0.0 & $163.8 \mathrm{~b}$ & $0.08 \mathrm{a}$ \\
\hline & 2000 & $272.3 \mathrm{~b}$ & $179.3 \mathrm{a}$ & $211.8 \mathrm{c}$ & 37975.7 b & 1900 & 0.0 & $179.3 \mathrm{a}$ & $0.09 a$ \\
\hline & 3000 & $3640 \mathrm{a}$ & $164.3 \mathrm{~b}$ & $283.0 \mathrm{a}$ & $46497.0 \mathrm{a}$ & $23.3 \mathrm{a}$ & 0.0 & $1643 \mathrm{~b}$ & $0.08 \mathrm{a}$ \\
\hline \multirow{5}{*}{$\begin{array}{l}\text { squash } \\
\text { (Tabarak F1) }\end{array}$} & 0 & 1130.800 & $404.0 \mathrm{a}$ & $152.3 \mathrm{C}$ & $61509.0 \mathrm{~b}$ & $30.8 \mathrm{~b}$ & $688.3 \mathrm{~b}$ & $1092.3 \mathrm{a}$ & $0.55 \mathrm{a}$ \\
\hline & 500 & $1962.8 \mathrm{~b}$ & $262.0 \mathrm{c}$ & $219.3 \mathrm{~b}$ & $57456.6 \mathrm{~b}$ & $28.7 \mathrm{~b}$ & 419.30 & $681.3 \mathrm{c}$ & $0.34 \mathrm{c}$ \\
\hline & 1000 & $2356.8 \mathrm{a}$ & 294.80 & $308.8 \mathrm{a}$ & $910342 \mathrm{a}$ & $45.5 \mathrm{a}$ & $553.3 \mathrm{C}$ & 848.00 & $0.42 \mathrm{D}$ \\
\hline & 2000 & 1074.50 & 143.30 & $314.3 \mathrm{a}$ & $450392 \mathrm{C}$ & $22.5 \mathrm{C}$ & $922.8 \mathrm{a}$ & $1066.0 \mathrm{a}$ & $0.53 \mathrm{a}$ \\
\hline & 3000 & $13090 \mathrm{c}$ & 119.30 & $251.0 \mathrm{D}$ & 29944.30 & 1500 & $167.0 \mathrm{e}$ & 286.30 & 0.140 \\
\hline \multirow{5}{*}{$\begin{array}{l}\text { Cow pea } \\
\text { (Qaha 1) }\end{array}$} & 0 & $432.3 \mathrm{a}$ & 275.0 a & $501.8 \mathrm{a}$ & $137995.0 \mathrm{a}$ & $69.0 \mathrm{a}$ & $198.0 \mathrm{~b}$ & $473.0 \mathrm{D}$ & $0.24 \mathrm{D}$ \\
\hline & 500 & $447.0 \mathrm{a}$ & $281.3 \mathrm{a}$ & $474.3 \mathrm{a}$ & $133420.6 \mathrm{a}$ & $66.7 \mathrm{a}$ & $1383 \mathrm{C}$ & $419.5 \mathrm{D}$ & $0.21 \mathrm{c}$ \\
\hline & 1000 & $324.3 \mathrm{~b}$ & 240.00 & $423.8 \mathrm{~b}$ & $1017120 \mathrm{~b}$ & $509 \mathrm{D}$ & $382.5 \mathrm{a}$ & $6225 \mathrm{a}$ & $0.31 \mathrm{a}$ \\
\hline & 2000 & $310.8 \mathrm{~b}$ & $126.0 \mathrm{c}$ & 404.80 & $752928 \mathrm{C}$ & $37.7 \mathrm{C}$ & $127.8 \mathrm{C}$ & $313.8 \mathrm{C}$ & 0.160 \\
\hline & 3000 & $265.3 \mathrm{C}$ & $187.3 \mathrm{C}$ & $329.3 \mathrm{C}$ & $61677.9 \mathrm{c}$ & $30.8 \mathrm{c}$ & $404.0 \mathrm{a}$ & $591.3 \mathrm{a}$ & $0.30 \mathrm{a}$ \\
\hline
\end{tabular}

In each column, values of a crp cu rivars folowed by the same letter(s) are not signiflcantly of frerent $(\mathrm{P}-0.05)$. 
Table (3): Development and reproduction of $R$. reniform is on some vegetable crops as affected by different concentrations of sodium chloride.

\begin{tabular}{|c|c|c|c|c|c|c|c|c|c|c|}
\hline \multirow{2}{*}{ Vegetable cr op } & \multirow{2}{*}{$\begin{array}{c}\text { Salt } \\
\text { concentration }\end{array}$} & \multicolumn{9}{|c|}{ Nematode counts } \\
\hline & & $\begin{array}{l}\text { D. } \$ 1 \\
\text { plant }\end{array}$ & $\begin{array}{l}\text { M.female5/ } \\
\text { plant }\end{array}$ & $\underset{\substack{\text { Eggma } \\
\text { lplant }}}{ }$ & $\begin{array}{c}\text { Eggs/ } \\
\text { eggmas } 5\end{array}$ & $\underset{50 \| l l}{E g g 5 / g}$ & $\begin{array}{c}\text { Total no. } \\
\text { eggs }\end{array}$ & $\begin{array}{c}\text { Soll } \\
\text { population }\end{array}$ & $\begin{array}{c}\text { Final } \\
\text { population }\end{array}$ & PTIPI \\
\hline \multirow{5}{*}{$\begin{array}{l}\text { Eggplant } \\
\text { (Gloya na) }\end{array}$} & 0 & $5.3 \mathrm{a}$ & $5.0 \mathrm{DC}$ & $140.0 \mathrm{a}$ & $80.3 \mathrm{a}$ & $5.6 \mathrm{a}$ & $11242.0 \mathrm{a}$ & $6340 \mathrm{C}$ & $7843 \mathrm{C}$ & 0.390 \\
\hline & 500 & $1.5 \mathrm{~b}$ & $4.3 \mathrm{C}$ & $150.3 \mathrm{a}$ & $73.0 \mathrm{a}$ & $5.5 \mathrm{a}$ & 109719 a & $1093.8 \mathrm{a}$ & $1249.8 \mathrm{a}$ & $0.62=$ \\
\hline & 1000 & 1.00 & $43 \mathrm{c}$ & 12000 & $83.0 \mathrm{a}$ & $5.0 \mathrm{a}$ & $99600 \mathrm{a}$ & $964.0 \mathrm{D}$ & $1089.3 \mathrm{~b}$ & 0.54 \\
\hline & 2000 & $5.5 \mathrm{a}$ & $6.8 \mathrm{~b}$ & $1098 \mathrm{~b}$ & $720 \mathrm{a}$ & 400 & $7905.6 \mathrm{~b}$ & $154.0 \mathrm{e}$ & $276.0 \mathrm{e}$ & $0.14 \mathrm{e}$ \\
\hline & 3000 & $3.3 \mathrm{ab}$ & $10.3 \mathrm{a}$ & $139.0 \mathrm{a}$ & $70.8 \mathrm{a}$ & 49 a & $98412 \mathrm{a}$ & 478.80 & 631.30 & 0.320 \\
\hline \multirow{5}{*}{$\begin{array}{l}\text { Squash } \\
\text { (Tabarak F1) }\end{array}$} & 0 & 5.30 & $9.0 \mathrm{a}$ & $6228 \mathrm{a}$ & $82.8 \mathrm{a}$ & $25.8 \mathrm{a}$ & $51567.8 \mathrm{a}$ & $3105.3 \mathrm{a}$ & $3742.3 \mathrm{a}$ & $1.87 \mathrm{a}$ \\
\hline & 500 & $8.0 \mathrm{a}$ & $10.8 \mathrm{a}$ & $276.8 \mathrm{C}$ & $77.0 \mathrm{a}$ & $10.7 \mathrm{~b}$ & $21313.6 \mathrm{~b}$ & $2644.0 \mathrm{D}$ & $2938.8 \mathrm{~b}$ & 1.47 \\
\hline & 1000 & $1.0 \mathrm{c}$ & 5.80 & $279.8 \mathrm{C}$ & 4480 & $6.3 \mathrm{c}$ & $12535.0 \mathrm{C}$ & $2640.0 \mathrm{D}$ & $2925.5 \mathrm{~b}$ & 1.46 \\
\hline & 2000 & $1.8 \mathrm{c}$ & $5.3 \mathrm{~b}$ & $441.3 \mathrm{D}$ & 5280 & $11.7 \mathrm{c}$ & $23300.6 \mathrm{~b}$ & $1573.5 \mathrm{c}$ & $2021.8 \mathrm{C}$ & 1.010 \\
\hline & 3000 & $3.8 \mathrm{~b}$ & 4.50 & $281.8 \mathrm{C}$ & $39.5 \mathrm{D}$ & $5.6 \mathrm{C}$ & $11131.1 \mathrm{C}$ & $2378.8 \mathrm{~b}$ & $2668.8 \mathrm{~b}$ & 1.33 \\
\hline \multirow{5}{*}{$\begin{array}{l}\text { Cowpea } \\
\text { (Qaha 1) }\end{array}$} & 0 & 5.30 & $13.5 \mathrm{~b}$ & $226.8 \mathrm{c}$ & $103.0 \mathrm{bc}$ & $11.7 \mathrm{c}$ & $23380.4 \mathrm{C}$ & $2724.0 \mathrm{~b}$ & $2969.5 \mathrm{~b}$ & 1.48 \\
\hline & 500 & $7.5 \mathrm{a}$ & $230 \mathrm{a}$ & 25880 & $1120 \mathrm{~b}$ & $14.5 \mathrm{~b}$ & $28985.6 \mathrm{~b}$ & $2666.8 \mathrm{bc}$ & $2956.0 \mathrm{~b}$ & 1.48 \\
\hline & 1000 & $3.0 \mathrm{c}$ & $6.8 \mathrm{c}$ & $316.3 \mathrm{a}$ & $135.8 \mathrm{a}$ & $21.5 \mathrm{a}$ & $42953.5 \mathrm{a}$ & 1904.30 & $2230.3 \mathrm{c}$ & 1.120 \\
\hline & 2000 & 5.00 & $9.3 \mathrm{c}$ & 17080 & 95.3 od & 8.10 & 1627720 & $2337.5 \mathrm{c}$ & $25225 \mathrm{c}$ & 1.260 \\
\hline & 3000 & $2.8 \mathrm{c}$ & 13.80 & 18200 & 8000 & 7.30 & 14560.50 & $3718.3 \mathrm{a}$ & $3916.8 \mathrm{a}$ & 196 \\
\hline
\end{tabular}

in each column, values of a crop cu rivars fo lowed by the same letter(s) are not signilicantly of ferent $(\mathrm{p}-0.05)$. 
Soil population behaved in different pattern, the lowest numbers of soil population were counted on eggplant comparing to the other tested hosts. The $500 \mathrm{ppm} \mathrm{NaCl}$ concentration achieved the highest significant soil population when compared to the check and other treatments. On squash, soil populations significantly decreased by all salt concentrations.

On the contrary, the highest soil population of the reniform nematode on cowpea was counted at the highest concentration of salt. Similar results were observed with the final population and the rate of nematode build up. It could be generally concluded that the influence of salt concentration on the development and reproduction on nematode depend on nematode species, salt concentration and soil EC as well as the cultivated host plant.

Data in tables $(4,5)$ illustrate the response of the host plants to nematode infection under the stress of salt. It is obvious, in the majority of cases, that salt stress significantly increased the damage caused by nematodes. The percentages of reduction in plant growth criteria were as follows: In case of $M$. incognita, 0.0 $25.2 \%$ in shoot length, $0-79.4 \%$ in shoot fresh weight, $14.9-82.4 \%$ in shoot dry weight, $0-41.0 \%$ in root length and $12.8-80.8 \%$ in root weight. The highest percentages of reduction were observed on eggplant. In case of $R$. reniformis, 0 $44.9,32.0-82.6,27.7-87.3,0-34.1$ and $25.2-85.9 \%$, respectively. It is clear that, damage in plants under the stress of salt and infected with the reniform nematode was more pronounced than those infected with the root-knot nematode. Such damage emphasized that the tested vegetables are sensitive to salt especially when the measured electrical conductivity of the soil ranged between $<1-3.74$ which ranked as low - slight saline soil.

\section{Discussion}

The effect of increasing salt concentrations in irrigation water was studied to clarify the role of salinity on the development and reproduction of the root-knot and reniform nematodes and the response of the cultivated vegetable crops. The present results are staggering whereas the influence of $\mathrm{NaCl}$ on the root-knot nematode M.incognita depended on salt concentration and the growing crop. On eggplant, increasing salt concentration resulted in significant increase in the number of galls, egg-masses, the number of eggs/g soil as well as the final population. Increasing $\mathrm{NaCl}$ concentration in irrigation water increased females fecundity on the roots of eggplant and squash, however, on cowpea roots the opposite was observed. The highest rates of build up were observed at $2000 \mathrm{ppm}$ of $\mathrm{NaCl}$ (EC. 3.16-3.21, 3.12-3.17) on eggplant and squash and at 1000 ppm on cowpea (EC. 1.88-2.13). Venkates and Das (1980) observed positive correlation between nematode population and chloride salts content of soil water. Kirkpatrick and VanGundy (1966) found that development of Tylenchulus semipenetrans increased by increasing salinity until $6.5 \mathrm{mmhos} / \mathrm{cm}$. Salinity increased egg production of 
Table (4): Growth of some vegetables as affected by M. incognita and NaClconcent ations.

\begin{tabular}{|c|c|c|c|c|c|c|c|c|c|c|c|c|}
\hline \multirow{2}{*}{ Host } & \multicolumn{6}{|c|}{ Shoot } & \multicolumn{4}{|c|}{ Root } & \multicolumn{2}{|c|}{ Total weight } \\
\hline & length & $\%$ red. & F. weight & $\%$ red. & D.weight & $\%$ red. & length & $\%$ red. & weight & $\%$ red. & weight & $\%$ red. \\
\hline \multicolumn{13}{|l|}{ Eggplant } \\
\hline Untreated & $26.00 \mathrm{~d}$ & & $6.31 \mathrm{a}$ & & $1.02 \mathrm{a}$ & & $28.38 \mathrm{a}$ & & $4.58 \mathrm{a}$ & & $10.89 a$ & \\
\hline 0 salt & $32.25 \mathrm{~b}$ & - & $2.63 \mathrm{~b}$ & 58.32 & $0.43 \mathrm{~b}$ & 57.84 & 20.25 bc & 22.21 & $2.48 \mathrm{~b}$ & 33.28 & $5.10 \mathrm{~b}$ & 53.17 \\
\hline $500 \mathrm{ppm}$ & 30.00 bc & - & $1.60 \mathrm{c}$ & 74.64 & $0.23 \mathrm{c}$ & 77.45 & $19.50 \mathrm{bc}$ & 31.13 & $2.28 \mathrm{c}$ & 50.21 & $3.88 \mathrm{c}$ & 62.87 \\
\hline $1000 \mathrm{ppm}$ & $28.00 \mathrm{~cd}$ & - & $1.50 \mathrm{c}$ & 76.23 & $0.18 \mathrm{c}$ & 82.35 & $19.38 \mathrm{bc}$ & 31.71 & $1.48 \mathrm{~d}$ & 57.69 & $2.98 \mathrm{~d}$ & 72.64 \\
\hline $2000 \mathrm{ppm}$ & $26.50 \mathrm{~d}$ & - & $1.30 \mathrm{c}$ & 79.40 & $0.18 \mathrm{c}$ & 82.35 & $19.38 \mathrm{bc}$ & 40.97 & $1.08 \mathrm{e}$ & 76.42 & $2.38 \mathrm{~d}$ & 78.15 \\
\hline $3000 \mathrm{ppm}$ & $35.25 \mathrm{a}$ & - & $1.50 \mathrm{c}$ & 76.23 & $0.18 \mathrm{c}$ & 82.35 & $21.75 b$ & 23.36 & $0.88 \mathrm{f}$ & 80.79 & $2.38 \mathrm{~d}$ & 78.15 \\
\hline \multicolumn{13}{|l|}{ Squash } \\
\hline Untreated & $46.40 \mathrm{a}$ & & $20.34 a$ & & $2.79 a$ & & $23.00 \mathrm{ab}$ & & $3.34 \mathrm{a}$ & & $23.68 \mathrm{a}$ & \\
\hline 0 salt & $34.73 d$ & 25.15 & $12.81 \mathrm{~b}$ & 37.02 & $1.93 \mathrm{~b}$ & 30.82 & 20.25 bc & 11.96 & $2.71 \mathrm{~b}$ & 18.86 & $15.52 \mathrm{~b}$ & 34.46 \\
\hline $500 \mathrm{ppm}$ & $37.58 \mathrm{~cd}$ & 19.01 & $8.87 \mathrm{c}$ & 56.46 & $1.18 \mathrm{c}$ & 57.71 & $28.50 \mathrm{a}$ & - & $2.93 \mathrm{~b}$ & 12.78 & $11.79 \mathrm{c}$ & 50.21 \\
\hline $1000 \mathrm{ppm}$ & $42.83 \mathrm{ab}$ & 7.69 & $7.48 \mathrm{~d}$ & 63.23 & $1.08 \mathrm{c}$ & 61.29 & 21.75 bc & 5.43 & $2.26 \mathrm{c}$ & 32.34 & $9.74 d$ & 54.69 \\
\hline $2000 \mathrm{ppm}$ & 40.33 bc & 13.08 & $6.30 \mathrm{e}$ & 69.03 & $0.88 \mathrm{~d}$ & 68.46 & $15.50 \mathrm{~d}$ & 32.61 & $1.76 \mathrm{~d}$ & 47.31 & $8.06 \mathrm{e}$ & 59.56 \\
\hline $3000 \mathrm{ppm}$ & $42.42 \mathrm{~b}$ & 8.56 & $5.96 \mathrm{e}$ & 70.69 & $0.86 \mathrm{~d}$ & 69.48 & $18.50 \propto d$ & 19.50 & $1.22 \mathrm{e}$ & 63.47 & $7.18 \mathrm{e}$ & 65.50 \\
\hline \multicolumn{13}{|l|}{ Cowpea } \\
\hline Untreated & $21.75 d$ & - & $7.50 \mathrm{a}$ & & $1.88 \mathrm{a}$ & & $20.50 \mathrm{~b}$ & & $5.88 \mathrm{a}$ & & $13.38 \mathrm{a}$ & \\
\hline 0 salt & $49.75 a$ & - & $7.93 \mathrm{a}$ & - & $1.60 \mathrm{~b}$ & 14.89 & $23.75 a b$ & - & $4.28 \mathrm{~b}$ & 27.21 & $12.20 \mathrm{~b}$ & 8.82 \\
\hline $500 \mathrm{ppm}$ & $32.38 \mathrm{c}$ & - & $5.88 \mathrm{~b}$ & 21.60 & $1.08 \mathrm{c}$ & 42.55 & $23.13 a b$ & - & $4.18 \mathrm{~b}$ & 28.41 & $10.06 \mathrm{c}$ & 15.55 \\
\hline $1000 \mathrm{ppm}$ & $37.95 b$ & - & $4.73 c$ & 36.93 & $0.60 \mathrm{~d}$ & 68.09 & $2425 a b$ & - & $3.00 \mathrm{c}$ & 48.98 & $7.73 d$ & 42.23 \\
\hline $2000 \mathrm{ppm}$ & $26.93 \mathrm{~cd}$ & - & $4.80 \mathrm{c}$ & 36.00 & $0.68 \mathrm{~d}$ & 63.83 & $27.00 \mathrm{a}$ & - & $2.43 \mathrm{c}$ & 58.67 & $7.23 \mathrm{~d}$ & 45.96 \\
\hline $3000 \mathrm{ppm}$ & $31.58 \mathrm{c}$ & - & $4.23 c$ & 43.60 & $0.73 d$ & 61.17 & $24.08 a b$ & - & $2.18 \mathrm{c}$ & 62.93 & $6.40 \mathrm{e}$ & 52.16 \\
\hline
\end{tabular}

In each column, values of a cop cu rivars followed by the same letter $(s)$ are not sgnilicantly of rerent $(\mathrm{P}-0.05)$. 
Al-Sayed A. Al-Sayed et al.,....................

Table (5): Growth $\alpha$ iteria of some vegetables as affected by $R$. reniform is and $\mathrm{NaCl}$ concentrations

\begin{tabular}{|c|c|c|c|c|c|c|c|c|c|c|c|c|}
\hline \multirow{2}{*}{ Host } & \multicolumn{6}{|c|}{ Shoot } & \multicolumn{4}{|c|}{ Root } & \multicolumn{2}{|c|}{ Total weight } \\
\hline & Length & \%red. & F. weight & Gred. & D.weight & Mred. & Length & $\%$ red. & Weight & Gred. & weight & $\%$ red. \\
\hline $\begin{array}{l}\text { Eggplant } \\
\text { Untreated } \\
0 \text { salt } \\
500 \mathrm{ppm} \\
1000 \mathrm{ppm} \\
2000 \mathrm{ppm} \\
3000 \mathrm{ppm}\end{array}$ & $\begin{array}{l}26.00 \mathrm{c} \\
31.25 \mathrm{~b} \\
29.63 \mathrm{~b} \\
31.00 \mathrm{~b} \\
36.25 \mathrm{a} \\
38.75 \mathrm{a} \\
\end{array}$ & $\begin{array}{l}- \\
- \\
- \\
\end{array}$ & $\begin{array}{l}6.31 \mathrm{a} \\
3.85 \mathrm{~b} \\
1.60 \mathrm{~d} \\
1.48 \mathrm{~d} \\
2.90 \mathrm{c} \\
2.53 \mathrm{c} \\
\end{array}$ & $\begin{array}{l}38.96 \\
74.84 \\
76.55 \\
54.04 \\
59.90 \\
\end{array}$ & $\begin{array}{l}1.02 \mathrm{a} \\
0.65 \mathrm{~b} \\
0.13 \mathrm{~d} \\
0.25 \mathrm{~cd} \\
0.28 \mathrm{c} \\
0.20 \mathrm{c}\end{array}$ & $\begin{array}{l}36.27 \\
87.25 \\
75.49 \\
72.55 \\
80.39 \\
\end{array}$ & $\begin{array}{l}28.25 \mathrm{a} \\
18.88 \mathrm{c} \\
18.63 \mathrm{c} \\
19.38 \mathrm{c} \\
22.63 \mathrm{~b} \\
23.25 \mathrm{~b}\end{array}$ & $\begin{array}{l}33.17 \\
34.05 \\
31.34 \\
19.89 \\
17.70 \\
\end{array}$ & $\begin{array}{l}4.58 \mathrm{a} \\
2.35 \mathrm{~b} \\
1.33 \mathrm{c} \\
0.90 \mathrm{~d} \\
0.73 \mathrm{e} \\
0.65 \mathrm{e}\end{array}$ & $\begin{array}{l}48.69 \\
70.96 \\
80.35 \\
84.06 \\
85.91\end{array}$ & $\begin{array}{c}10.89 \mathrm{a} \\
6.02 \mathrm{~b} \\
2.93 \mathrm{~cd} \\
2.38 \mathrm{e} \\
3.63 \mathrm{c} \\
3.25 \mathrm{od}\end{array}$ & $\begin{array}{l}56.57 \\
73.09 \\
78.15 \\
66.67 \\
70.16 \\
\end{array}$ \\
\hline $\begin{array}{l}\text { Squash } \\
\text { Untreated } \\
0 \text { salt } \\
500 \mathrm{ppm} \\
1000 \mathrm{ppm} \\
2000 \mathrm{ppm} \\
3000 \mathrm{ppm} \\
\end{array}$ & $\begin{array}{l}46.40 \mathrm{a} \\
27.43 \mathrm{c} \\
25.55 \mathrm{c} \\
33.65 \mathrm{~b} \\
26.05 \mathrm{c} \\
29.00 \mathrm{c} \\
\end{array}$ & $\begin{array}{l}40.88 \\
44.94 \\
27.48 \\
43.86 \\
37.50 \\
\end{array}$ & $\begin{array}{c}20.34 \mathrm{a} \\
13.03 \mathrm{~b} \\
4.98 \mathrm{c} \\
4.28 \mathrm{~cd} \\
3.55 \mathrm{~d} \\
3.60 \mathrm{~d}\end{array}$ & $\begin{array}{l}35.94 \\
78.96 \\
75.52 \\
82.55 \\
82.30 \\
\end{array}$ & $\begin{array}{l}2.79 \mathrm{a} \\
1.83 \mathrm{~b} \\
0.60 \mathrm{c} \\
0.58 \mathrm{c} \\
0.55 \mathrm{c} \\
0.57 \mathrm{c} \\
\end{array}$ & $\begin{array}{l}34.41 \\
78.49 \\
79.21 \\
80.29 \\
79.57 \\
\end{array}$ & $\begin{array}{l}23.00 \mathrm{bc} \\
22.13 \mathrm{c} \\
24.00 \mathrm{bc} \\
25.30 \mathrm{~b} \\
25.38 \mathrm{~b} \\
30.50 \mathrm{a}\end{array}$ & $\begin{array}{l}3.78 \\
- \\
- \\
- \\
\end{array}$ & $\begin{array}{l}3.34 \mathrm{a} \\
2.50 \mathrm{~b} \\
0.95 \mathrm{~d} \\
1.25 \propto d \\
1.28 \mathrm{c} \\
1.23 \propto d \\
\end{array}$ & $\begin{array}{l}25.15 \\
71.56 \\
62.57 \\
61.68 \\
63.17 \\
\end{array}$ & $\begin{array}{r}23.68 \mathrm{a} \\
12.53 \mathrm{~b} \\
5.93 \mathrm{c} \\
5.53 \mathrm{c} \\
4.83 \mathrm{c} \\
4.83 \mathrm{c} \\
\end{array}$ & $\begin{array}{l}45.52 \\
74.22 \\
75.96 \\
79.00 \\
79.00 \\
\end{array}$ \\
\hline $\begin{array}{l}\text { Cowpea } \\
\text { Untreated } \\
0 \text { salt } \\
500 \mathrm{ppm} \\
1000 \mathrm{ppm} \\
2000 \mathrm{ppm} \\
3000 \mathrm{ppm}\end{array}$ & $\begin{array}{l}21.75 \mathrm{~b} \\
27.58 \mathrm{a} \\
25.70 \mathrm{ab} \\
24.68 \mathrm{ab} \\
25.33 \mathrm{ab} \\
23.70 \mathrm{ab}\end{array}$ & $\begin{array}{l}- \\
- \\
-\end{array}$ & $\begin{array}{l}7.50 \mathrm{a} \\
5.10 \mathrm{~b} \\
4.00 \mathrm{~cd} \\
4.80 \mathrm{bc} \\
3.63 \mathrm{~d} \\
4.00 \mathrm{~cd}\end{array}$ & $\begin{array}{l}32.00 \\
46.67 \\
36.00 \\
51.60 \\
46.67\end{array}$ & $\begin{array}{l}1.88 \mathrm{a} \\
1.36 \mathrm{~b} \\
0.60 \mathrm{c} \\
0.53 \mathrm{c} \\
0.50 \mathrm{c} \\
0.53 \mathrm{c}\end{array}$ & $\begin{array}{l}27.68 \\
68.09 \\
71.81 \\
73.40 \\
71.81\end{array}$ & $\begin{array}{l}20.50 a b \\
23.25 a b \\
20.38 b \\
23.75 a \\
20.00 b \\
20.75 a b\end{array}$ & $\begin{array}{l}\overline{0} .59 \\
\bar{z} \\
2.44\end{array}$ & $\begin{array}{l}5.83 \mathrm{a} \\
3.25 \mathrm{~b} \\
1.18 \mathrm{c} \\
1.30 \mathrm{c} \\
1.40 \mathrm{c} \\
1.00 \mathrm{c}\end{array}$ & $\begin{array}{l}44.25 \\
79.76 \\
77.70 \\
75.99 \\
82.84\end{array}$ & $\begin{array}{r}13.33 \mathrm{a} \\
8.35 \mathrm{~b} \\
5.18 \mathrm{~d} \\
6.10 \mathrm{c} \\
5.03 \mathrm{~d} \\
5.00 \mathrm{~d}\end{array}$ & $\begin{array}{l}37.36 \\
61.14 \\
54.24 \\
62.27 \\
62.49\end{array}$ \\
\hline
\end{tabular}

In each column, values of a crop cu rivars folowed by the same letter( $(8)$ are not sgnilicantly orferent $(\mathrm{p}-0.05)$. 
T. semipenetrans by 2-10 folds when $\mathrm{NaCl}$ was added in irrigation water every other day (Mashela et al., 1992). Other research workers observed reduction in nematode activities as a result of increasing salt concentrations. Sweelam (1994) found that gall numbers caused by Meloidogyne javanica on tomato roots were reduced by increasing sodium chloride concentrations from 1000 to $4000 \mathrm{ppm}$. Vermak et al., (2007) as well, stated that galling, egg-masses, eggs/egg-mass and final population in the soil decreased with increasing EC. levels from 5.6-11.2 as compared to non-saline (EC. 1.15).

With the reniform nematode, the opposite was observed, whereas nematode counts increased with increasing salt concentration on cowpea and decreased on eggplant and squash. But, the results emphasized that damage of plants under the stress of salt and the reniform nematode infection was more pronounced than those infected with the root-knot nematode. Heald and Heilman (1971) stated that the reniform nematode occurred equally in relatively low saline $(4.0 \mathrm{mmhos})$ and highly saline $(16.5 \mathrm{mmhos} / \mathrm{cm})$ soils. Generally, limits of nematode tolerance to salt concentration varied to great extent according to nematode genera and/or species. Many nematode species tolerate slightly saline conditions (EC. 2-4 mmhos/cm) e.g Tylenchus, Tylenchorhynchus, Pratylenchus and Aphelenchoides. Helicotylencus dihystera, Hirshmanniela gracilis and Macropsthonia ornata thrived moderately saline soils with ECs around $6 \mathrm{mmhos} / \mathrm{cm}$. Few other species like Rotylenchulus reniformis, Meloidogyne sphaerocephala, Hemicriconemoides cocophilus and Caloosia exitis tolerate saline soil conditions (EC. $10.25 \mathrm{mmhos} / \mathrm{cm}$ ); Ray and Das, 1980. The nematode activities affected by salt concentrations are egg hatching (Dropkin et al., 1958; Lal and Yadav, 1975), invasion of plant roots (Wallace, 1970), Development (Kirkpatrick and Van Gundy, 1966; Youssef et al., 1989), reproduction (Edongali and Ferris, 1980) and survival (Jairajpuri et al., 1974).

The present results also indicated that slightly saline soils adversely affected the growth of plants especially vegetables. These results coincide with those of Vermak et al.(2007) and Karajeh and Al-Nasir (2008).

\section{References}

Dropkin, V.H.; Martin, C.C. and Johnson, R.W. (1958). Effect of osmotic concentration on hatching of some plant parasitic nematodes. Nematologica, 3: 115-126.

Edongali, F.A.; Duncan, L. and Ferris, H. (1982). Influence of salt concentration on infectivity and development of $M$. incognita on tomato. Revue Nematology, 5 (1): 111-117.

Edongali, E.A. and Ferris, H. (1980). Varietal response of tomato to salinityMeloidogyne incognita interaction. Journal of Nematology, 12 (4): 218. 
Edongali, F.A. and Ferris, H. (1981). Effect of soil salinity and temperature on reproduction and egg hatching of $M$. incognita in tomato. Nematologica Mediterranea, 9 (2): 123-132.

Heald, C.M. and Heilman, M.D. (1971). Interaction of Rotylenchulus reniformis, soil salinity, and cotton. Journal of Nematology, 3 (2): 179-182.

Jairajpuri, M.Sh.; Azmi, M.J. and Bajaj, H.K. (1974). Effect of pH and salt concentration on the survival of nematode, Hoplolaimus indicus, Helicotylenchus indicus, Xiphinema basiri and Mylonchulus minor. Indian Journal of Nematology, 4:171-181.

Karajeh, M.R. and AI-Nasir, F.M. (2008). Salt suppression of Meloidogyne javanica on tomato. Nematologia Mediterranea, $36: 185-190$.

Khan, A.A. and Khan, M.W. (1990). Influence of salinity stresses on hatching and juvenile mortality of root-knot nematodes, Meloidogyne incognita (race 2) and Meloidogyne javanica. Pakistan Journal of Nematology, 8 (2): 107111.

Kirkpatrick, J.D. and Van Gundy, S.D. (1966). Soil salinity and citrus nematode survival. Nematologica, 12 : 93-94 (Abstr.).

Lal, A. and Yadav, B.S. (1975). Effect of leaches from saline soils on hatching of eggs of $M$. incognita Indian Journal of Nematology, 5(2): 228-229.

Mashela, P; Duncan, L. and McSorley, R. (1992). Salinity resuces resistance to Tylenchulus semipenetrans in citrus rootstocks. Nematropica, 22 (1): 712.

Ray, S. and Das, S.N. (1980). Nematode of saline soils in Orissa, India. Indian Journal of Nematology, 10(2): 231-260.

SPSS (2003). Statistical package for the social science Incorporation. Chicago, SPSS base 12 application guide. Chicago: SPSS, X1 426 pp.

Sweelam, M.E. (1994). Sensitivity of root-knot nematode Meloidogyne javanica to Gamma irradiation, salinity and plant growth regulator, cycocel. Second Arab Conference on the Peaceful Uses of Atomic Energy, Cairo, 5-9 November 1994: 926-931.

Venkates, W.G. and Das, R.M. (1980). Studies on the correlation of benthic nematode populations with hardness of water. Proceedings of the Indian Academy of parasitology, 1 (2): 129-131.

Vermak, K.K.; Jain, R.K. and Sharma, S.K. (2007). Influence of soil salinity on reproduction and multiplication of root-knot nematode, (Meloidogyne incognita) in cotton. Indian Journal of Nematology, 37(1).

Wallace, H. R. (1970). Some factors influencing nematode reproduction and the growth of tomatoes infected with M. javanica. Nematologica 16:387-397.

Youssef, G.M.; Farahat, A.A. and Elnagar, H.I. (1989). Reproduction of the citrus nematode, Tylenchulus semipenetrans as influenced by chloride salts. Bullten Faculty Of Agriculture, Cairo University, 40 (2): 515-526. 


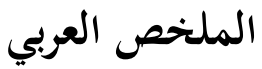

تأثير ملوحة مياه الري على القدرة الإمراضية وتكاثر نيماتودا تعقد الجذور والنيماتودا الكلوية على بعض نباتات الخضر

السيد أبو المعاطي السيد، أحمد عبد السلام فرحات ، شيماء فتحي دياب قسم الحيوان والنيماتولوجيا الزراعية - كلية الزراعة - جامعة القاهرة

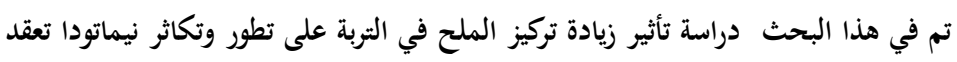

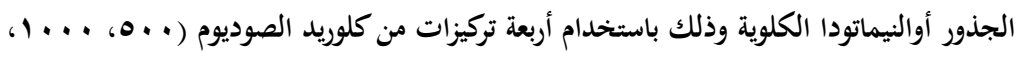

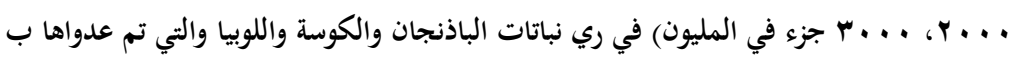

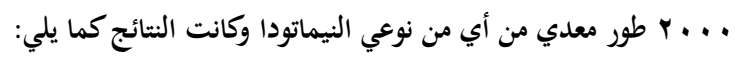

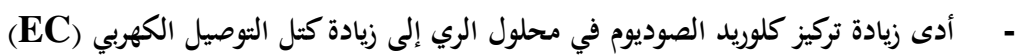

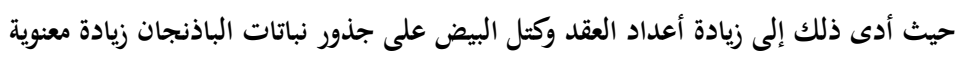

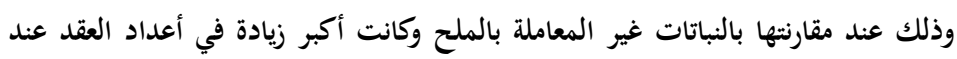

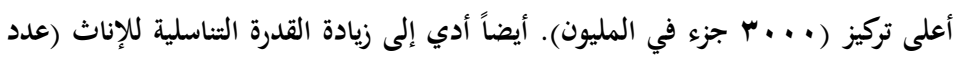

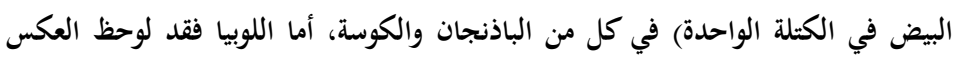

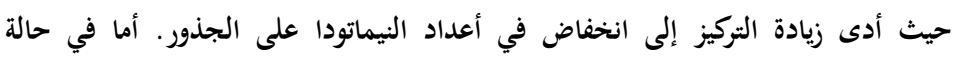

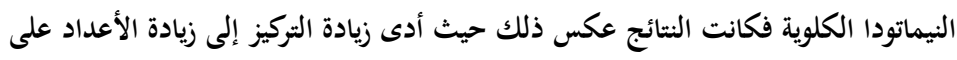
جذور اللوبيا ونقصها على جذور الباذنجان والكوسة.

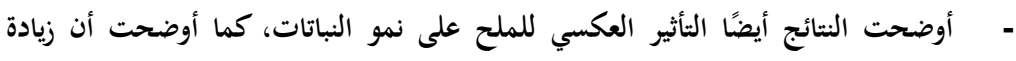

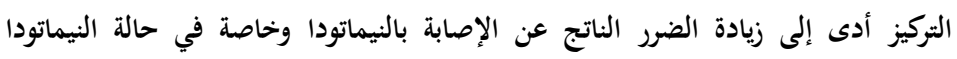

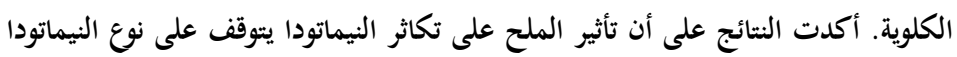

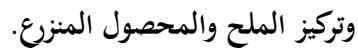

